\title{
Detection and Treatment Systems based on Optics and Photonics in New Age Healthcare Management
}

\section{Gaurav Pandey ${ }^{1}$, Rashmi Chaudhari² and Abhijeet Joshi*1}

${ }^{1}$ Centre for Biosciences and Biomedical Engineering, Indian Institute of Technology Indore, India

${ }^{2}$ Department of Biosciences and Bio-Engineering, Indian Institute of Technology Bombay, India

\section{Editorial}

The world is becoming smaller in the means of communication and information but we are yet short to make basic medical needs deliverable universally. There have been many breakthroughs and advancements in the field of medical and healthcare but they fall scant in stature, in terms of taking care of major healthcare problems across the globe. If we look closely, major healthcare problems can be classified as primary and secondary. Accurate diagnosis, treatment and therapies for severe diseases and disorders which are prolonged threat to life form the primary healthcare problems. Secondary health care problems include conditions like trauma, or accidents, or mishaps in daily life. The latter healthcare conditions demand for quick and immediate medical attention for stabilizing the victims and then initiate recovery. Human population is advancing in terms of medical technologies with respect to diagnostic and treatment modalities to tackle serious healthcare issues, but most of the latest medical technologies are available in established centres which are formed by a high economic investment. The high cost makes the technology unavailable to a majority of the population residing in developing countries. In cases of road trauma several factors need to be considered within a small time frame to provide immediate medical supervision, also medical procedures to be provided simultaneously in no time. The loss in time in such cases eventually leads to loss of life or loss of functionality of organs. Excessive losses of blood or internal injuries are most common but are involved with most time consumption for proper attention and diagnosis leading to irreversible lethality to important organs like brain, heart, liver or kidneys. Thus researchers are continuously developing diagnostic and therapeutic systems which are affordable in resource poor settings. Optical technologies provide most suitable approach for deployment in resource poor settings. Optical technologies are continuously evolving and advancing our healthcare systems. Proper utilization and conditioning of light spectrum and exploring optical properties of novel materials have shown promising results for development of medical techniques. Modification, assembly and combination of optical equipment's can be easily integrated to form dedicated instruments for health anomalies. As a requirement to increase deliverability, solutions need to rely on new components which travel and transfer information faster. Use of light for above purpose can solve all the above mentioned perils and also provide noninvasive and harmless method of diagnosis and treatment. Diagnostic and scanning techniques like PET, X-ray, CT etc. have already employed optical techniques for in vivo imaging and surgery. Endoscopic examination of GIT involves use of light and camera inserted using a flexible tube and a colour TV monitor is the basic example of use of optics for imaging and visualization of disease manifestations [1]. On the other hand, lasers are part of helpful optical instruments in the medical sector from deep vein thrombosis (DVT) to sutures and vessels sealing from time to time [2,3] In case of DVT by 'Laser Thrombolysis' (Use of laser pulses to remove the clots) using different sets of lasers and intensity, timings are used to disintegrate the clot without making unnecessary incisions [2-4]. Lasers are also deployed in sensitive surgical procedures for vision impairment owing to its precision, reach, and less invasive properties. Apart from proven aesthetic uses like tattoo and pigmentation removal, optical instruments are also to be utilized in the field of non-invasive patient monitoring systems as optical pulse and oxygen saturation analyser for uninterrupted patient recovery and observation. Various skin diseases are being tested by scope of optical scanning including skin cancer, chronic pain using 'photo-dynamic therapy' $[2,3]$. The scope of optical techniques in diagnostic and therapeutic usage has been described in the adjoining (Figure 1). The use of optics is not restricted to one property or one field but it appears as vast as its extension in spectrum thus the same source and property of light can also be used in other objectives with few changes, establishing its integration compactly and low energy tool developments. These prospects are greatest and most desirable cues for using optical technology in almost every field where portability and ambulatory utilization is very high in demand. Recently, a healthcare company 'THERANOS' developed 140 types of testing and analysis with a single drop of blood and has been well received in scientific community [5]. The product signifies the precision, reaching ability, less invasive nature and adaptability to different needs of the system. Similarly, optical characteristic profiling gives strong hope for testing other soft or hard tissue like bone and density and bodily samples as well on the basis of signal processing internally or by sampling them out in very fewer quantities than the existing procedures. In another application of pulse oximetry red and infra-red light is utilized to get the absorbance of dissolved gasses and saturation level of biological carriers like haemoglobin. Continuous monitoring systems like pulse oximetry capable to detect pulse rates, $\mathrm{O}_{2}$ and $\mathrm{CO}_{2}$ gasses saturation level can be deployed [1-2,6-12]. Infrared thermometers or pulse laser thermometers or temperature guns working on the principle of capturing and measuring infrared emittance of a heated body by focusing it to a particular spot; which is not only applicable in medical field but serves very vast field of applications and industrial areas. Lasers and optics make another robust base for telemedicine and remote analysis prospective in healthcare field with appropriate placements and counter check with other indicative and suggestive parameters. Telemedicine and remote analysis can provide a huge possibility for screening by non-invasive method and remote execution. Development of analytic tools in movement and body structure related disorders with added benefit of being non-invasive as in GAIT and posture analysis [2-7]. Different types of lasers are being combined for

${ }^{*}$ Corresponding author: Abhijeet Joshi, Inspire Faculty, Centre for Biosciences and Biomedical Engineering, Indian Institute of Technology Indore, India; E-mail: abhijeet.joshi@iiti.ac.in

Received June 14, 2016; Accepted June 15, 2016; Published June 17, 2016

Citation: Pandey G, Chaudhari R, Joshi A (2016) Detection and Treatment Systems based on Optics and Photonics in New Age Healthcare Management. J Laser Opt Photonics 3: e110. doi:10.4172/2469-410X.1000e110

Copyright: @ 2016 Pandey G, et al. This is an open-access article distributed under the terms of the Creative Commons Attribution License, which permits unrestricted use, distribution, and reproduction in any medium, provided the original author and source are credited. 


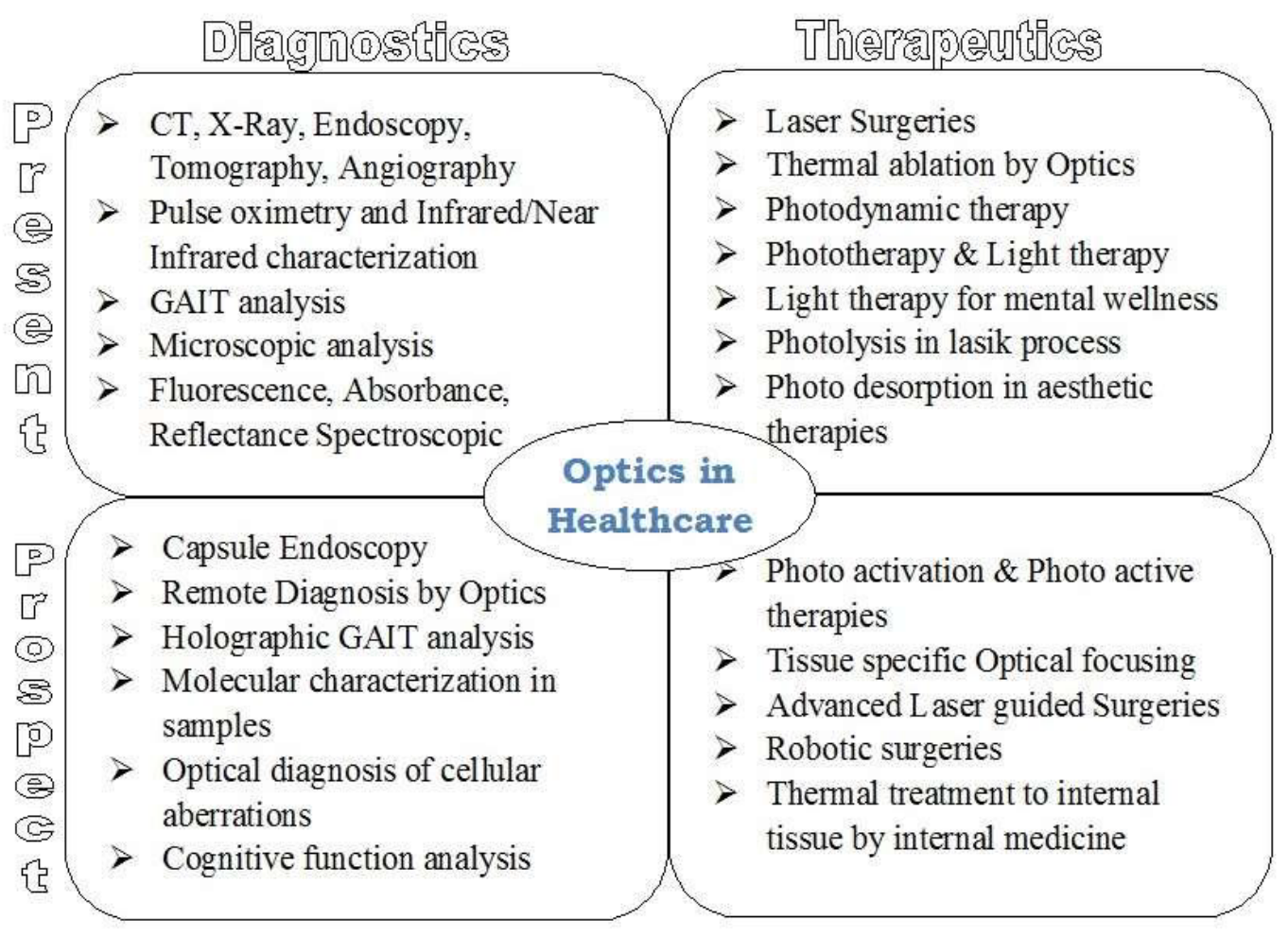

Figure 1: Scope of optics in diagnostics and therapeutics.

the therapies like $\mathrm{CO}_{2}$ lasers are used to make incisions in tissues with low vascularity (mostly used in neurosurgery for precision), while on the other hand with Nd-YAG (neodymium-doped yttrium aluminium garnet; Nd:Y3Al5O12) lasers are applicable in high vascular areas and tumour shrinkage, or surgical removal without leaving scars owing to energy dependent concentration where tumours can be thermally destroyed by concentrating the laser beam. This strategy also prevents undue toxicity to normal cells in comparison to other chemotherapeutic methods [2]. It also allows separation of tumours from vital structures minimizing low risk to surrounding tissues in the removal of tumours with surgeries. Use of monochromatic light absorption property different levels of tissues within internal layers can be operated with success $[3,4]$. Another example is maintenance of secretory activity of small glands glands can be maintained by using a catheter to remove over-secretory regions by flashing laser pulses. Above mentioned properties surely give an upper hand to practitioners and surgeons for performing highly complex procedures in critical areas like brain where collateral damage can lead to severe implications. Needless to mention that these procedures save a lot of time, effort, risk of collateral damage to surrounding tissues, and manage blood loss in comparison to normal surgical procedure subsequently leading to early and sound recovery without any scar formation or associated complications. Conditions associated with brain are most susceptible and irreversible to collateral damage owing to non-repairable nature of brain structures. Cerebral hypoxia associated with trauma cases due to low blood pressure or clot formation and subsequent fall in essential nutrition is one such process which is not completely understood by researchers and physicians. The neurons are dependent for supplies on the blood flow to the brain and other parts of nervous system, and these constant supplies are utilized in the energy production to carry out different duties, most primarily conduction of impulses via interneuron junctions called synapse in a vast interconnected complex network lying all across the brain tissue. Whenever brain is exposed to resource scanty conditions the important physiological responses are hampered as brain doesn't have any kind of storage facility for energy produced. The brain does not have pain receptors or physical warning signs for indicating ongoing damage which ultimately leads to brain tissue death [1,2-15]. Cerebral hypoxia causes a partial/complete loss of functionality of brain tissues due to degeneration [12-18]. The most alarming part is that the function of that part cannot be restored by any present therapeutic manner as brain tissue doesn't inhabit the power of regeneration, surgical and chemotherapeutic treatments are also less effective due to complexity of the organ and the presence of blood brain barrier. Invasive procedures for brain trauma treatment are associated with vulnerabilities to damage of other parts of brain. In response to detrimental conditions, at times the brain activates and alters different control mechanisms of normal physiological factors and bodily conditions under its command; towards the values, which are of opposite effect on present danger in order to nullify its further negative effect on neural tissue. The Brain raises cerebral blood pressure and breathing rates by neural control centre to fulfil elevated demand of oxygen or nutrient. Along with these parameters neural activity at brain also increases several folds to prepare and release more counter measures for present dangerous situation, as biochemical messengers responsible to initiate vascularisation and elevate blood pressure at cellular level by constricting blood vessels, are released in bloodstream returning from cerebral region to increase blood vessels [11,14,15-17]. We believe that research in mapping of cerebral hypoxia can be a new 
Citation: Pandey G, Chaudhari R, Joshi A (2016) Detection and Treatment Systems based on Optics and Photonics in New Age Healthcare Management. J Laser Opt Photonics 3: e110. doi:10.4172/2469-410X.1000e110

Page 3 of 3

prospective area of research to be dealt using optical methodologies. It is also interesting to see the enthusiasm of national and international organizations who are supporting this initiative by financial and resources aid. It will be astonishing if in near future we can develop a whole automated setup based on optical and electrical components capable to capture whole body scans at once without any human intervention especially in remote and resource poor healthcare settings [3,5,19].

\section{References}

1. Yichao T, Haishu D, Qingcheng G (2005) In Non-invasive monitoring of human cerebral oxygen saturation by near infrared spectroscopy instrumentation calibration and application in cardiopulmonary bypass. IEEE Engineering in Medicine and Biology 27th Annual Conference, pp: 670-673.

2. Mendez A (2010) Medical Applications of Fiber-Optics Optical fiber sees growth as medical sensors.

3. Sathyam US, Shearin A, Prahl SA (1997) In Basic ablation phenomena during laser thrombolysis. pp: 19-27.

4. Beck OJ (1984) Use of the Nd-YAG laser in neurosurgery. Neurosurgical review 7: 151-157.

5. The Lab Test reinvented.

6. Bonnie F, Suzanne R, Lareau RN, Marianna S (2002) Pulse oximetry Principles of Pulse Oximetry Technology.

7. Principles of Pulse Oximetry Technology.

8. Battisti CA, Fisher J, Duffin J (2011) The cerebrovascular response to carbon dioxide in humans. Journal of Physiology-London 589: 3039-3048.

9. Belardinelli E, Ursino M, Digiammarco P (1988) A General-Model of Cerebrovascular Controls. Proceedings of the Annual International Conference of the leee Engineering in Medicine and Biology Society Pts, pp: 525-527.

10. Kresh JY, McVey MJ, Frasch HF, Pelleg A (1991) Brockman SK FrequencyDomain Analysis Of Arterial Blood-Pressure - A Probe For Central-NervousSystem. Activity IEEE, pp: 377-380.
11. Muthuswamy J, Sherman DL, Hinich MJ, Hanley DF, Thakor NV, et al. (1998) Spectral correlation of action potential firing rates after hypoxic-ischemic brain injury. In Proceedings of the 20th Annual International Conference of the leee Engineering in Medicine and Biology Society, 20: 2020-2022.

12. Zhang D, Ding H, Hou X, Liu Y, Ye D, et al. (2008) Cerebral hypoxic ischemia at different cerebral oxygen saturations in piglets amplitude-integrated EEG study. Conference proceedings Annual International Conference of the IEEE Engineering in Medicine and Biology Society. IEEE Engineering in Medicine and Biology Society. Annual Conference, pp: 4712-4715.

13. Dubinsky JM, Rothman SM (1991) Intracellular calcium concentrations during "chemical hypoxia" and excitotoxic neuronal injury. The Journal of neuroscience the official journal of the Society for Neuroscience 11: 2545-51.

14. Fabian RH, Perez P, Kent TA (2004) Extracellular superoxide concentration increases following cerebral hypoxia but does not affect cerebral blood flow. International Journal of Developmental Neuroscience, 22: 225-230.

15. Lovering AT, Dunin B, Vidruk W, Orem EH (2002) Neural control of airflow profile during hypoxia in sleep and wakefulness. In Second Joint Embs-Bmes Conference, pp: 1513-1514.

16. Caruso ALG, Bouillon TW, Schumacher PM, Luginbijhl M, Morari M, et al. (2007) Drug-induced respiratory depression an integrated model of drug effects on the hypersonic and hypoxic drive. In Annual International Conference of the leee Engineering in Medicine and Biology Society, pp: 4259-4263.

17. Li H, Zheng S, Zhang Z, Jiang D (2010) In Expression of Hypoxia-Inducible Factor in the Cerebral Ischemia/Reperfusion Injury Bioinformatics and Biomedical Engineering. International Conference, pp: 1-4.

18. Thakor NV, Kong X, Hanley DF (1995) Nonlinear changes in brain's response in the event of injury as detected by adaptive coherence estimation of evoked potentials. IEEE transactions on bio-medical engineering, 42: 42-51.

19. Rovati L, Fabbri P, Pilati F (2009) In Development of a low-cost pH sensor based on plastic optical fibers Instrumentation and Measurement. Technology Conference, pp: 1662-1665. 